\title{
Study and Evaluation of Advanced TOFD Method for Inspection of Polyethylene Pipes but Welding
}

\author{
Mohammad H. TAGHIPOUR \\ Pars Leading Inspection Company, Tehran, 18947, Iran
}

\begin{abstract}
Polyethylene material has some specification that makes very difficult any kind of inspection based on ultrasonic. The acoustic impedance and sound velocity in this kind of material are near to the materials commonly used in ultrasonic wedges. Also this kind of materials are highly attenuative materials for ultrasound. To inspection of polyethylene circumferential but welds and overcome to all the problems mentioned, the especial technique of TOFD (Time of Flight Diffraction) method which employs low frequency probe and concentrate on the inspection area that longitudinal waves turn into transverse waves has been used. The purpose of inspection is determining the exact location of surface and internal welding defects. For this purpose, two separate polyethylene pipes with 10 inch in diameter, $15 \mathrm{~mm}$ thickness and also 25 inch diameter, $28 \mathrm{~mm}$ thickness, were selected. In total 40 artificial defects which involve 28 side drill holes in deferent depths and 12 surface and sub-surface notches were created. All artificially created defects were detected with very good accuracy. Unlike the conventional TOFD method which have 2 to $3 \mathrm{~mm}$ dead zone, by using the above method even surface notch with $0.5 \mathrm{~mm}$ has been detected.
\end{abstract}

Key words: PE (Polyethylene pipe), ultrasonic, transverse wave, TOFD (Time of flight diffraction).

\section{Introduction}

The use of PE pipes for gas and fluid pipeline transport network nearly started about 1960 in the world and many countries started to apply their gas pipeline networks with PE pipes because of its benefits [1]. Very good resistance against breakage and cracking, excellent chemical resistance, corrosion resistance [2], lightness and ease of implementation of pipeline features are some benefits of PE pipes [3, 4]. The life time of PE pipeline is depend on proper operation of the welding and inspection operations to ensure full quality of polyethylene pipe welding's [5]. Up to now inspection of PE pipeline welding due to lack of reliable and verified method is limited to visual inspection [3]. However, the TOFD (Time of Flight Diffraction) inspection technique due to its unique characteristic can be used for PE (Polyethylene pipe) welding inspection. The TOFD inspection Technique in steels is known as the best method for sizing and

Corresponding author: Mohammad H. TAGHIPOUR, advanced NDT consultant, research field: engineering application of TOFD inspection. accuracy detection of defects $[4,6]$. In this paper the ability of TOFD defect detection in PE pipes were investigated and the capability of method for sizing defects requiring further investigation.

\section{Description}

Recently, some research and articles have been published by some international companies such as Olympus and TWI about applying TOFD and Phased Array method for inspection of Welds in PE pipes. This publications are including the use of some special equipments like water wedge and low frequency TOFD and Phased Array probe in addition of main equipments [3, 4]. This special equipment is to overcome the problems facing these methods for inspecting PE pipes. The properties such as high attenuation of ultrasound waves in polyethylene materials and having similar acoustic impedance and velocity of ultrasonic wave with the materials normally using for TOFD and Phased Array probe shoes [3, 6]. This properties of PE materials, according to Snell's low causes the ultrasound waves cannot completely 
pass throw the probe wedge to the PE specimen $[6,7]$.

Typically the TOFD inspection technique is concentrated in an area between the waves from the surface (Lateral Wave) and from back wall (Back wall echo) in steel materials [6]. Fig. 1 shows an overview of the TOFD technique.

But always after the waves from the back wall, the waves are due to changing nature of longitudinal waves to transverse waves (Mode conversion) that can be configured and monitor on the device along with other waves [6].

This study suggests that unlike steel materials in polyethylene materials the mode conversion area could help to detection of defects and there is no need to use special water wedge.

To investigate the ability of TOFD method for detection of defects in deferent depths, two separate polyethylene pipes with 10 inch in diameter, $15 \mathrm{~mm}$ thickness and also 25 inch diameter, $28 \mathrm{~mm}$ thickness, were selected. Totally 40 artificial defects which involve 28 side drill holes in deferent depths and 12 surface notches were created.

In the case of 10 inch pipe with $15 \mathrm{~mm}$ thickness, 16 side drilled hole with different depth and also 5 notches in upper surface and lower surface were created. Fig. 2 is scan of artificial defects from 1 to 5 which involve notches on upper and lower surface of specimen.

All five notches are well recognize in mode conversion area but the defect 3 which is grove in inner surface of the sample is detectable in the main area between lateral and close to the back wall.

Fig. 3 is a scan of defects from 7 to 15 , including the side drilled holes with depths in the range of 2 to 14 mms. The defects 12, 13 and 14, 15 are holes in a same
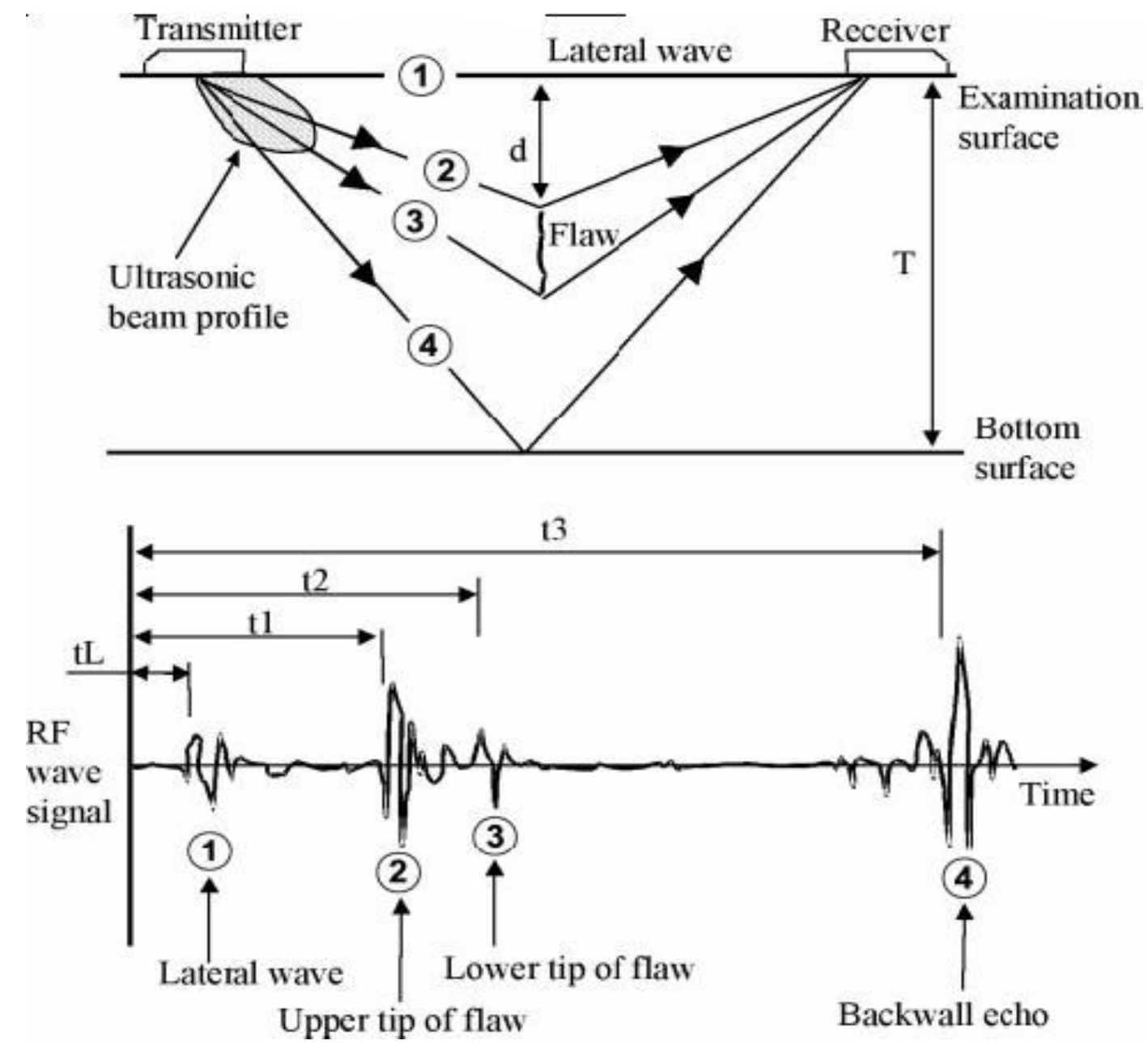

Fig. 1 Schematic of TOFD method represents A-Scan signals. 


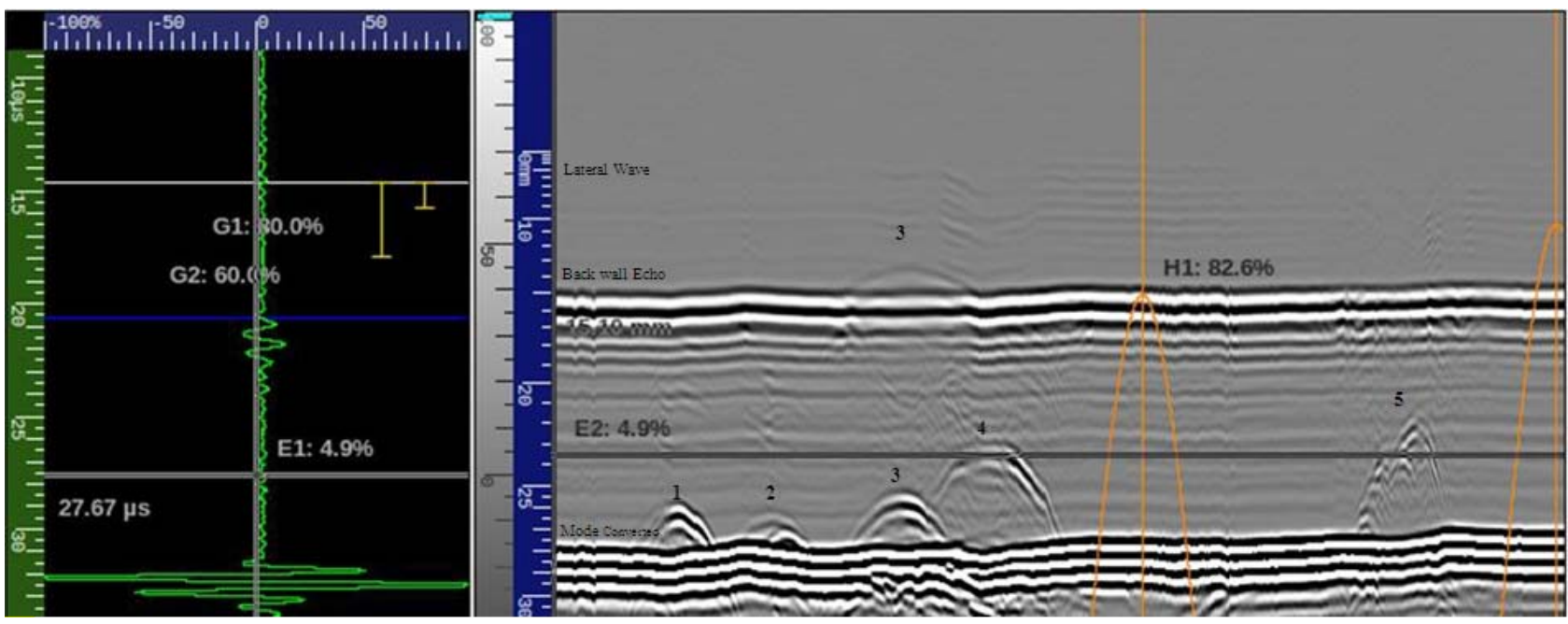

Fig. 2 Scan of artificial defects from 1 to 5 involve five notches.

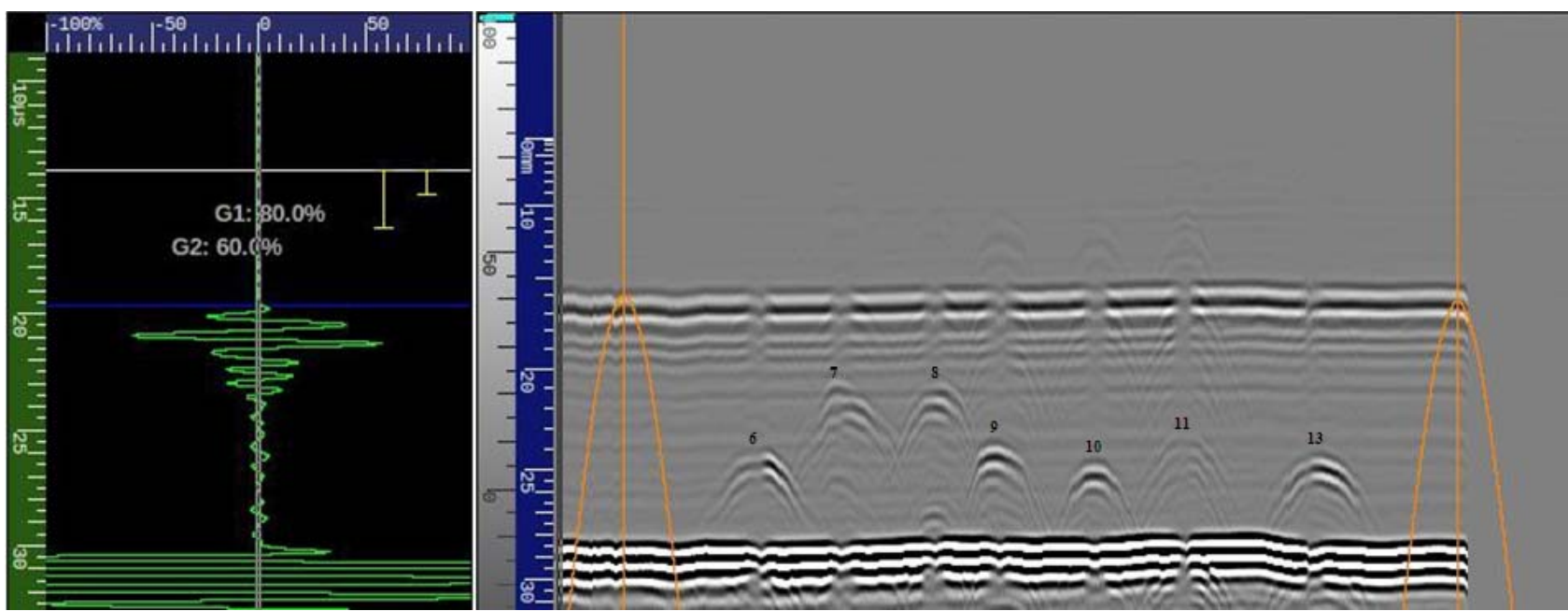

Fig. 3 Scan of artificial defects from 7 to 15 , including nine side drilled holes.

position with different depths.

In this case, it can be seen that from 9 side drilled hole only 7 holes are visible. It shows, from two defects at the same position with different depths, one of theme is not detectable. For this purpose in the scan file, the energy of the sound increase up to $20 \mathrm{~dB}$ shows in Fig. 4.

As shown in Fig. 4, all artificial defects are detectable in both main area and mode conversation.

Fig. 5 is a scan of defects from 16 to 19 which are side drilled holes respectively with depths of 12.5, 10.5, 10.7 and $9 \mathrm{~mm}$. It is obvious that all of these artificial defects are visible in both areas.

Fig.6 shows the scan of defects 19 to 22 which are side drilled holes respectively with depths of 9, 7, 4, and $1 \mathrm{~mm}$. All of artificial defects are detectable in both of inspection area.

The important point of this scan is, the defect number 22 is the side drilled hole with $1 \mathrm{~mm}$ depth from upper surface of sample. It is well detected in mode conversion area. Normally this kind of surface indications because of dead zone are not detectable in main area between lateral and back wall.

However, in this study the focus is on the detection of defects and not sizing of indications, in Fig. 7 tried to measuring the depth of indication 19 by TOFD method. The real depth of indication is $9 \mathrm{~mm}$.

Fig. 7 shows that the depth of indication in TOFD technique is $9.4 \mathrm{~mm}$. The difference from real depth is only $0.4 \mathrm{~mm}$. 

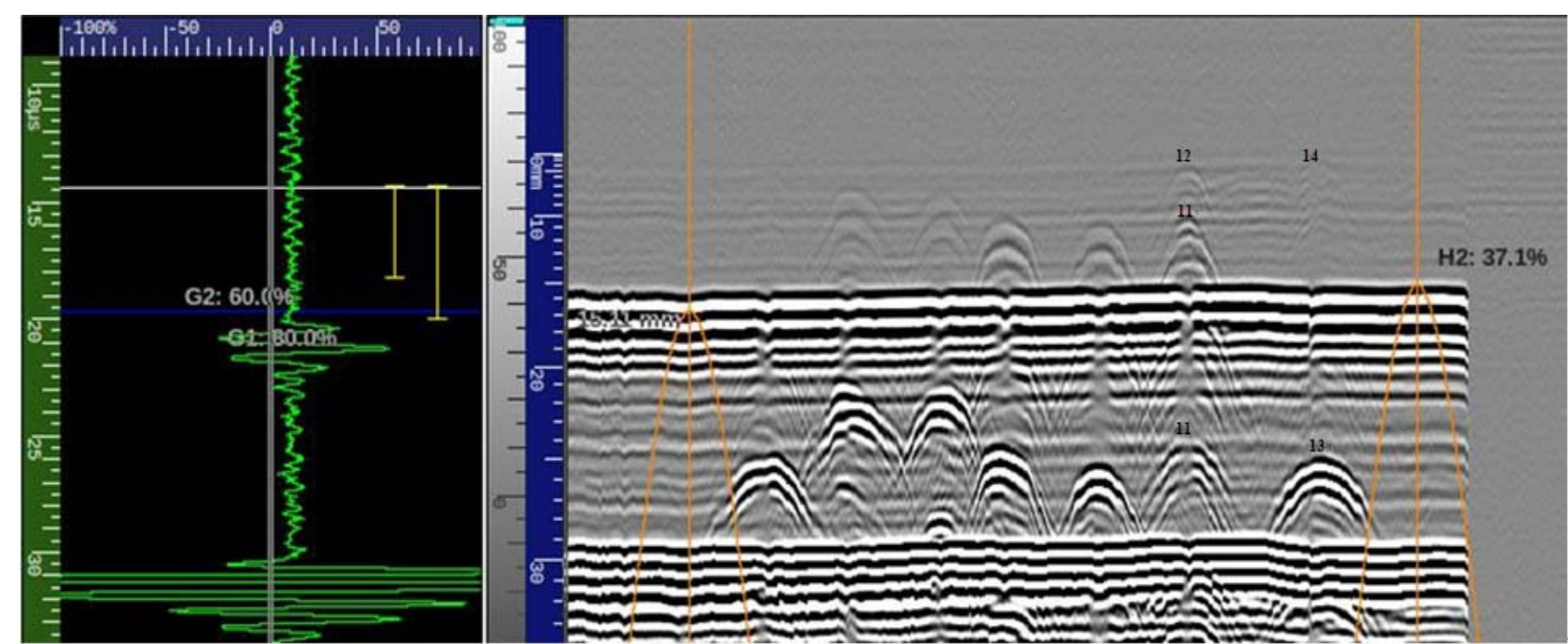

Fig. 4 Scan of artificial defects from 7 to 15 with increasing ultrasound energy up to $20 \mathrm{~dB}$.

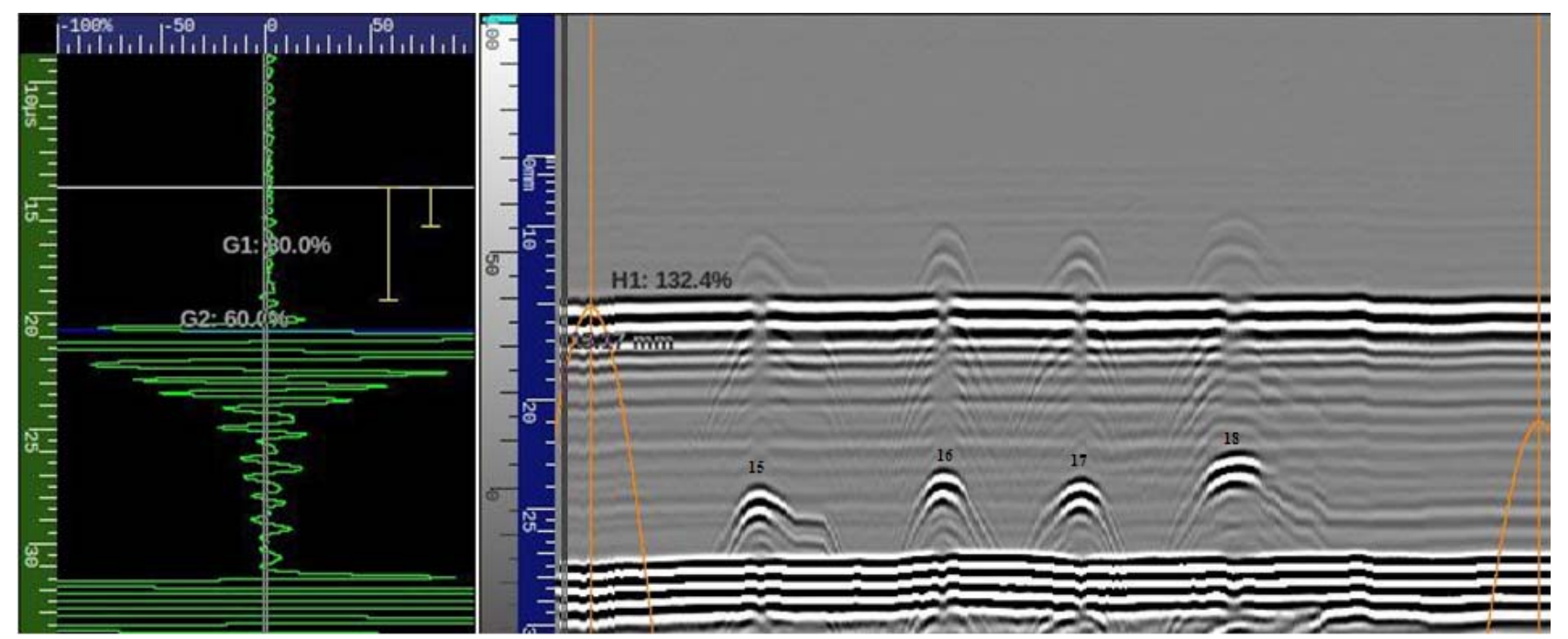

Fig. 5 Scan of artificial defects from 16 to 19 involve four side drilled holes.

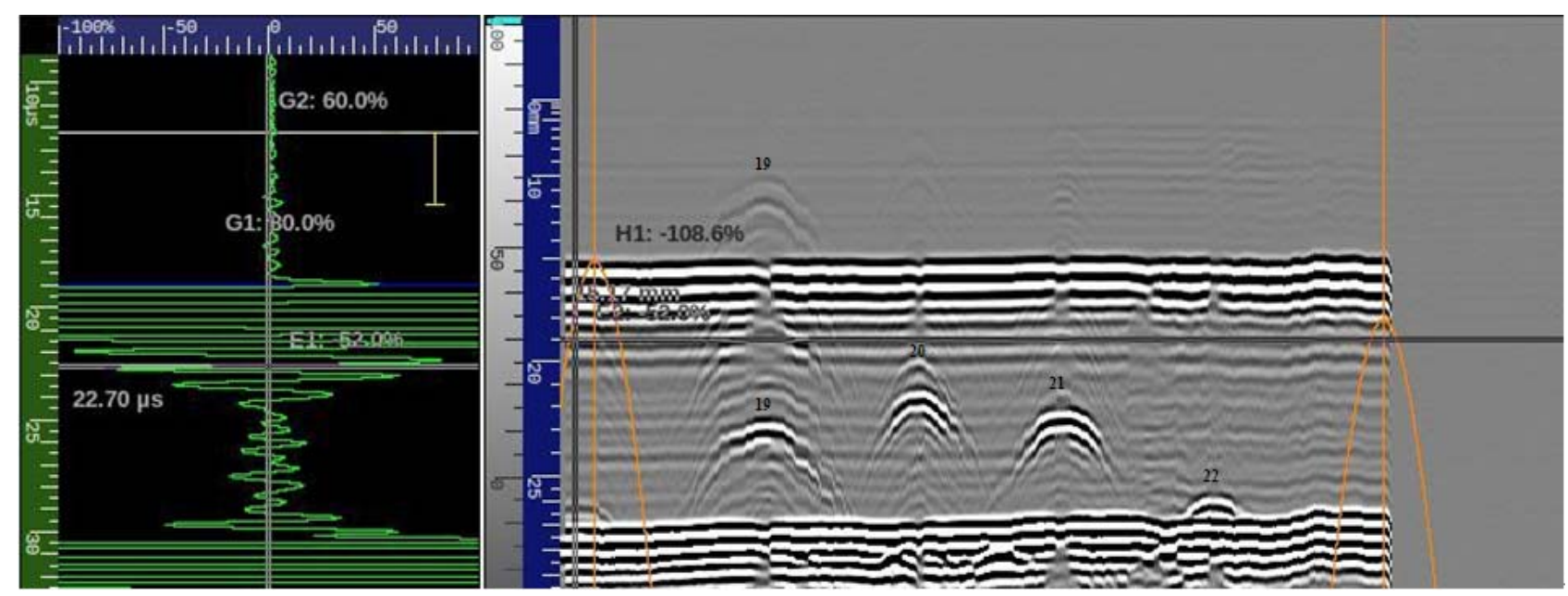

Fig. 6 Scan of artificial defects from 19 to 22 involve four side drilled holes. 

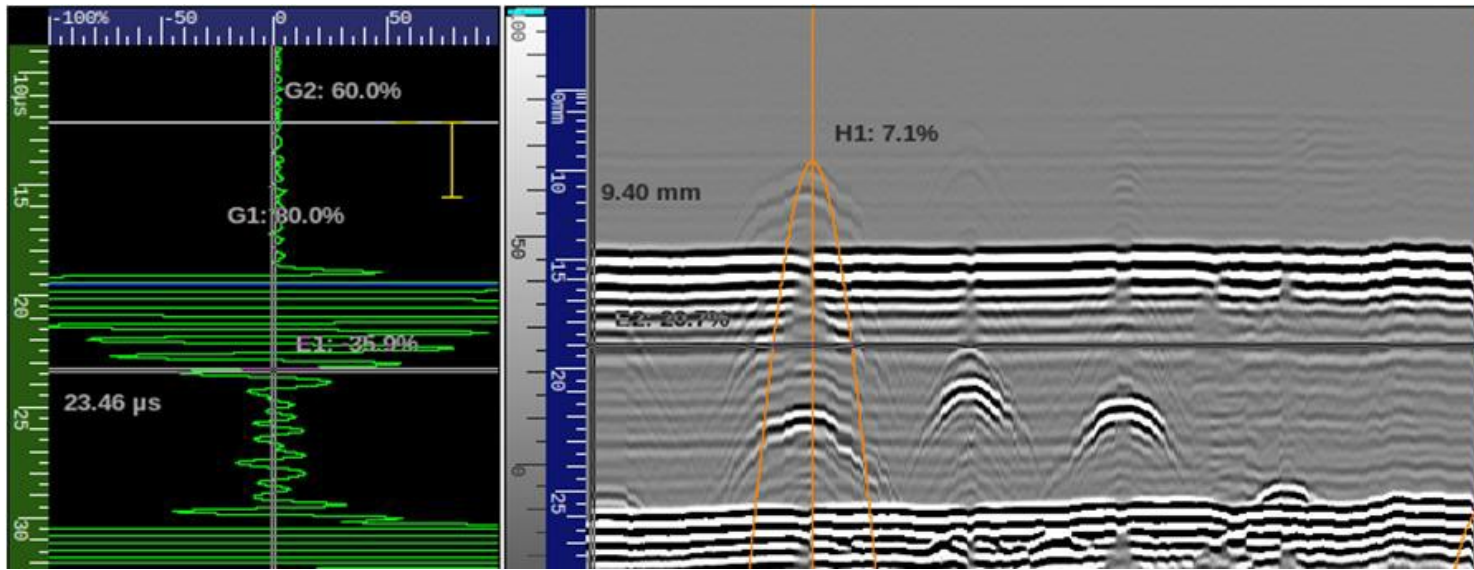

Fig. 7 Depth measuring of indication 19 by TOFD method.

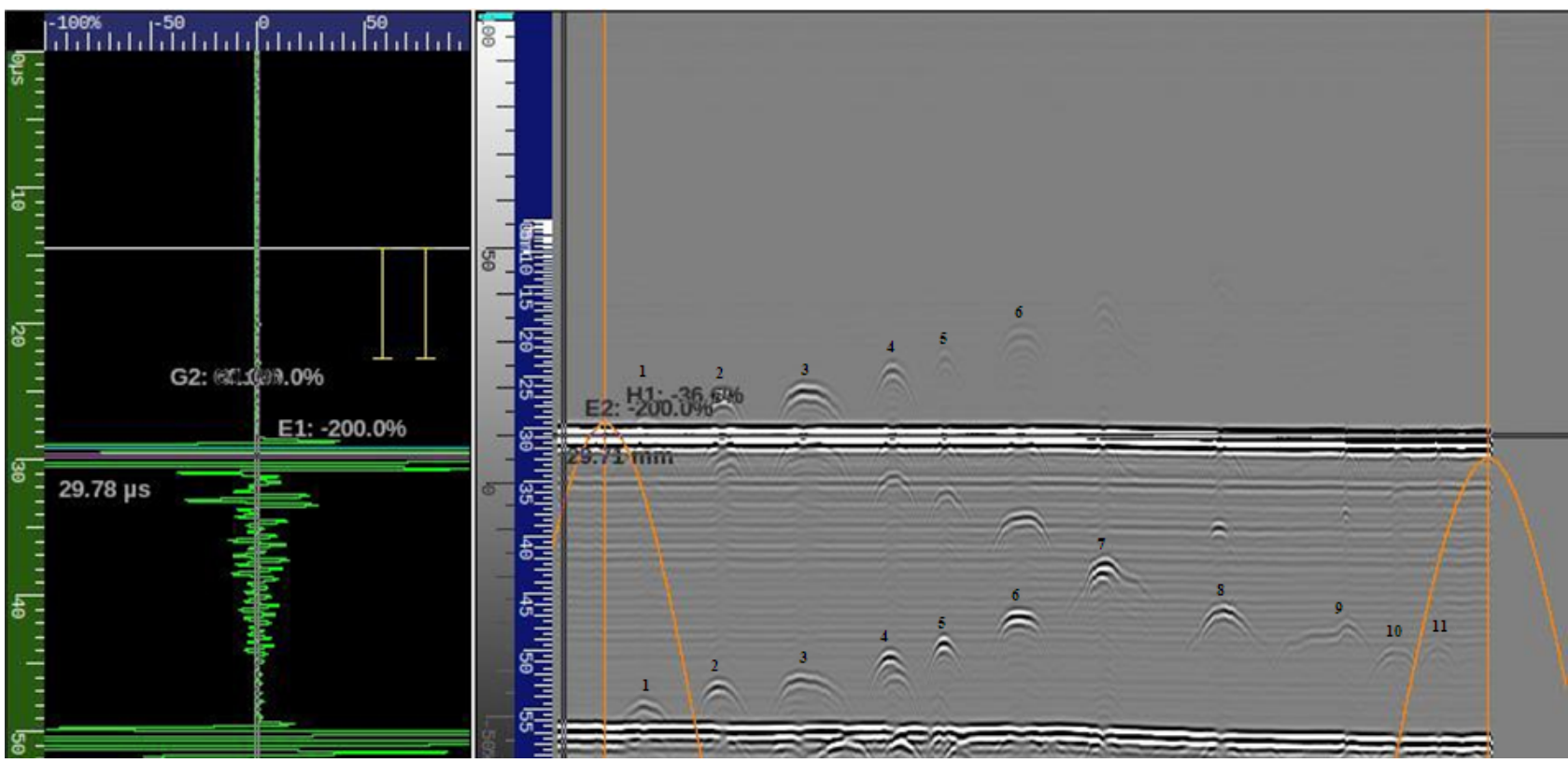

Fig. 8 Scan of artificial defects from first to 11 involve eleven side drilled holes in case of 25 inch pipe.
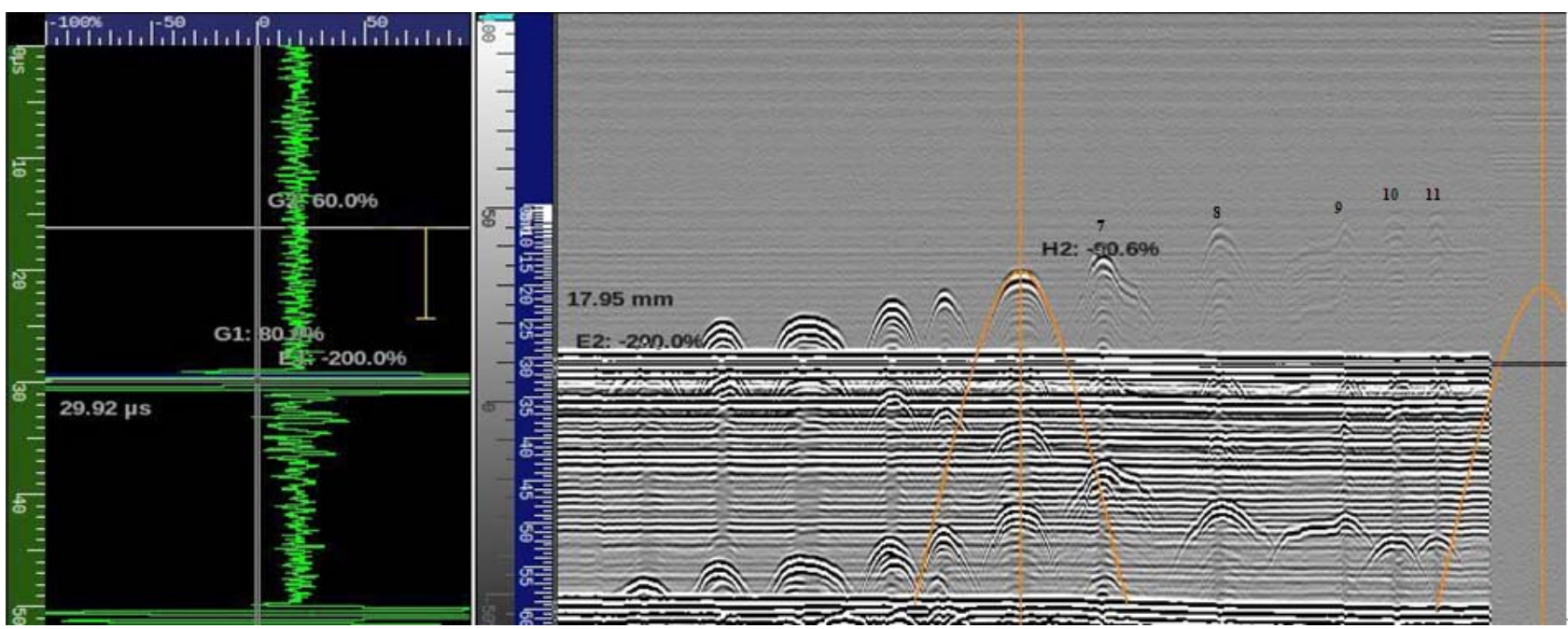

Fig. 9 Scan of artificial defects from first to 11 with increasing ultrasound energy up to $15 \mathrm{~dB}$. 


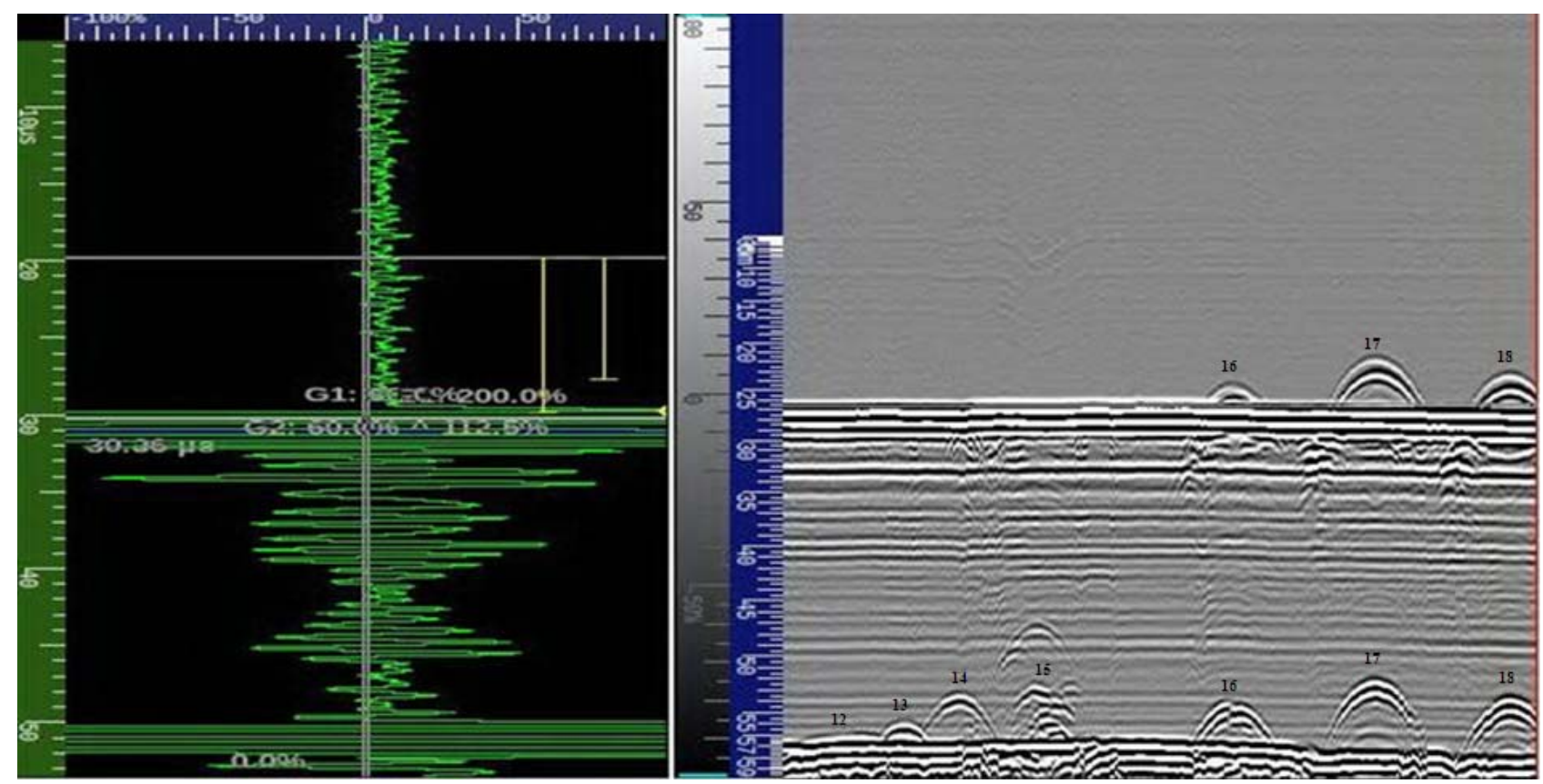

Fig. 10 Scan of artificial defects from 12 to 18 involve seven outer \& inner notches.

In the case of 25 inch pipe with $28 \mathrm{~mm}$ thickness, 11 side drilled holes with different depth from $26 \mathrm{~mm}$ to $1.5 \mathrm{~mm}$ and also 7 notches in upper surface and lower surface were created. Fig. 8 is scan of artificial defects from first to 11 which involve 11 side drilled holes with different depths.

According to the Fig. 8 all 11 artificial defects are well detectable in mode conversion area. Also the defects which are near to the inner surface of sample are well detectable in main inspection area between lateral and back wall. The defects near to the outer surface of sample are less detectable in main inspection area between lateral and back wall. To overcome this problem and identify all defects in both of scan area especially between lateral and back wall, in the scan file, the energy of the sound increase up to $15 \mathrm{db}$ which is shown in Fig. 9.

According to the Fig. 9, all artificial defects are detectable in both scan area. Also measuring depth of defect Number 6 with real $17 \mathrm{~mm}$ depth is $17.95 \mathrm{~mm}$, so the difference from actual depth is $0.95 \mathrm{~mm}$.

Fig. 10 shows scan of artificial defects from number 12 to 18 in 25 inch pipe sample.

This artificial defects are involve outer and inner surface notches respectively with $0.5,1,2,3.5,4,6$, $5 \mathrm{~mm}$.

According to the Fig. 10, all artificial notches are well detectable in mode conversion area. Also, the artificial notches which are near to the inner surface of sample are well detectable in main inspection area between lateral and back wall. The notches near to the outer surface of sample are not detectable in main inspection area between lateral and back wall.

\section{Conclusions}

According to the study carried out, all artificial defects involves side drilled holes in different depths and notches in inner and outer surface of samples were well detected in mode conversion area. The outer notches on the samples were detected only in mode conversion area and the inner notches on the samples were detected in both inspection area.

All side drilled holes in different depth on the samples were detected in both inspection area. For the full implementation of the study results with actual conditions in future studies, we will attempt with actual defects in welded polyethylene pipes for further evaluation. 


\section{References}

[1] PIPA TN016. “Non Destructive Examination of PE Welds Emerging Techniques IplexPoliplex Polyethylene Pipe Design Textbook.”

[2] Alex, S. and Doug, M. 2014. "Use of High Density Polyethylene for Repairs or Replacements of Buried Pipe." IAEA/EPRI Technical Meeting on Ageing Management of Buried and Underground Piping and Tanks for NPPs. October 13-15, Charlotte, NC.

[3] Hagglund, F. "Detection Capabilities of a Phased Array Ultrasonic Inspection System for Plastic Pipe Butt Fusion Joint.” Malcolm A Spicer and Mike J Troughton TWI Ltd.

[4] Mike, T., Malcolm, S. and Fredrik, H. 2012. "Development of Ultrasonic Phased Array Inspection of
Polyethylene Pipe Joints.’' PVP Pressure Vessel and Piping Conference.

[5] Frederick, C., Zimmerman, D. and Porter, A. 2009. "High-Density Polyethylene Pipingbutt-Fusion Joint Examination Using Ultrasonic Phased Array.” Journal of Pressure Vessels and Piping 7: 26-30.

[6] Crawford, S. L. and Cumblidge, S. E. 2008. "Technical Letter Report Preliminary Assessment of NDE Methods on Inspection of HDPE Butt Fusion Piping Joints for Lack of Fusion.” PNNL-17584.

[7] MacLennan, D., Pettigrew, I. G. and Bird, C. R. 2012. "Plastic Fantastic? An NDE Inspection Solution for HDPE Butt Welds." In Proceeding of 18th World Conference in Non-Destructive Testing, 16-20. Durban, South Africa. 